\title{
Planning and Budgeting for Nutrition Programs in Tanzania: Lessons Learned From the National Vitamin A Supplementation Program
}

\author{
Margaret Benjamin Lyatuu ${ }^{1 *}$, Temina Mkumbwa ${ }^{1}$, Raz Stevenson $^{2}$, Marissa Isidro $^{1}$, Francis Modaha ${ }^{3}$, Heather \\ Katcher ${ }^{1}$, Christina Nyhus Dhillon ${ }^{1}$
}

\begin{abstract}
Background: Micronutrient deficiency in Tanzania is a significant public health problem, with vitamin A deficiency (VAD) affecting 34\% of children aged 6 to 59 months. Since 2007, development partners have worked closely to advocate for the inclusion of twice-yearly vitamin A supplementation and deworming (VASD) activities with budgets at the subnational level, where funding and implementation occur. As part of the advocacy work, a VASD planning and budgeting tool (PBT) was developed and is used by district officials to justify allocation of funds. Helen Keller International (HKI) and the Tanzania Food and Nutrition Centre (TFNC) conduct reviews of VASD funds and health budgets annually in all districts to monitor the impact of advocacy efforts. This paper presents the findings of the fiscal year (FY) 2010 district budget annual review. The review was intended to answer the following questions regarding district-level funding: (1) how many funds were allocated to nutrition-specific activities in FY 2010? (2) how many funds were allocated specifically to twice-yearly VASD activities in FY 2010? and (3) how have VASD funding allocations changed over time?

Methods: Budgets from all 133 districts in Tanzania were accessed, reviewed and documented to identify line item funds allocated for VASD and other nutrition activities in FY 2010. Retrospective data from prior annual reviews for VASD were used to track trends in funding. The data were collected using specific data forms and then transcribed into an excel spreadsheet for analysis.

Results: The total funds allocated in Tanzania's districts in FY 2010 amounted to US\$1.4 million of which 92\% were for VASD. Allocations for VASD increased from US\$0.387 million to US\$1.3 million between FY 2005 and FY 2010. Twelve different nutrition activities were identified in budgets across the 133 districts. Despite the increased trend, the percentage of districts allocating sufficient funds to implement VAS (as defined by cost per child) was just $21 \%$. Discussion: District-driven VAS funding in Tanzania continues to be allocated by districts consistently, although adequacy of funding is a concern. However, regular administrative data point to fairly high and consistent coverage rates for VAS across the country (over $80 \%$ over the last 10 years). Although this analysis may have omitted some nutrition-specific funding not identified in district budget data, it represents a reliable reflection of the nutrition funding landscape in FY 2010. For this year, total district nutrition allocations add up to only $2 \%$ of the amount needed to implement nutrition services at scale according to Tanzania's National Nutrition Strategy Implementation Plan.

Conclusion: VASD advocacy and planning support at the district level has succeeded in ensuring district allocations for the program. To promote sustainable implementation of other nutrition interventions in Tanzania, more funds must be allocated and guidance must be accompanied by tools that enable planning and budgeting at the district level.

Keywords: Budget, Planning, Nutrition, Tanzania, Vitamin A Supplementation and Deworming (VASD)

Copyright: (C) 2016 by Kerman University of Medical Sciences

Citation: Lyatuu MB, Mkumbwa T, Stevenson R, et al. Planning and budgeting for nutrition programs in Tanzania: lessons learned from the national vitamin A supplementation program. Int J Health Policy Manag. 2016;5(10):583588. doi:10.15171/ijhpm.2016.46
\end{abstract}

\section{Article History:}

Received: 11 September 2015

Accepted: 23 April 2016

ePublished: 3 May 2016

\section{Key Messages}

Implications for policy makers

Based on the lessons learned from the vitamin A supplementation and deworming (VASD) program documented here:

- Standardized instruments are necessary to ensure realistic and justifiable budget allocations for identified interventions.

- Technical assistance in planning and costing not just implementing is a critical input to achieving acceptable programmatic achievements.

Implications for the public

This assessment demonstrates that some nutrition interventions like vitamin A supplementation and deworming (VASD) can be achieved at scale and institutionalised into subnational planning and budgeting systems, given strong advocacy and costing tools. A systematic review of district plans and budgets can also strengthen health systems and improve accountability at the local level, which is where nutrition budgets are allocated and spent in a country with a decentralized government like Tanzania. Monitoring activities like the one presented in this paper are an integral part of broader process evaluations to understand program effectiveness. 


\section{Background}

The Government of Tanzania signed on to the scaling up nutrition (SUN) movement in June 2011 establishing a commitment to improving nutrition in the country. In order to effectively scale up nutrition, the Prime Minister's Office prioritized three areas for SUN in Tanzania including the "translation of priority nutrition objectives into planned activities and budget allocations on an annual basis." Other priority areas included improving capacity for nutrition at regional and district levels. Alongside the SUN commitments in 2011, the Tanzania National Nutrition Strategy (20092015) Implementation Plan was finalized, endorsed and costed at just over US\$515 million, (approximately US\$103 million per year). Of this amount, roughly US\$331 million, or over US\$66 million per year, was estimated as the funding necessary to implement direct nutrition services ${ }^{[1]}$. In Tanzania's decentralized government, nutrition services are the responsibility of district health departments, and therefore, planned and budgeted in the annual district health plans, known as the Comprehensive Council Health Plans (CCHP). ${ }^{2}$ CCHPs are funded from government grants, donor health basket funds (HBFs), the districts' own resources and non-governmental organizations. ${ }^{2}$

One of the only successful nutrition programs implemented at scale in Tanzania is the twice-yearly national vitamin A supplementation and deworming (VASD) program. With consistently high reported, coverage rates since 2001 (Figure 1), the distribution and coverage of vitamin A supplementation has been cited as a contributing factor in the decline in the under-five mortality rate (U5MR) of children in the last decade. ${ }^{3}$ Currently Tanzania's VASD program reached approximately 6.8 million children twice a year.

The process for institutionalization of VASD as a scaled-up nutrition intervention in the country was carefully monitored and shepherded by VAS stakeholders between 2005 and 2008. In August 2001, Helen Keller International (HKI), with support from the United States Agency for International Development (USAID) and the United Nations Children's Emergency Fund (UNICEF), together with the Tanzania Food and Nutrition Centre (TFNC), began providing technical support, advocacy, and facilitation to all districts with the aim of ensuring a sufficient budget allocation for VASD in all district budgets.
This advocacy and facilitation work was implemented twiceyearly through 8 zonal-level and 21 regional-level planning, budgeting, monitoring, and review workshops held at the beginning of the annual budgeting cycle and aimed at council and district health management team members including VASD focal persons and planning/finance officers. Instrumental to this advocacy and facilitation work was a planning and budgeting tool (PBT) for VASD, shown in Figure 2, which details the necessary delivery activities with standard costing values and standardized population targets to help district planning teams arrive at a realistic and justifiable budget for VASD programs. The summarised calculations obtained are then transferred into the CCHP. In addition, districts exhibiting low coverage rates after implementation were given additional support to identify weaknesses in their planning and budgeting efforts by using a sustainability assessment tool developed by USAID/A2Z project. These workshops and tools were implemented in all districts across the country, beginning in 2007 with USAID support and continuing from 2010 to-date with support from the Canadian government (DFATD).

This paper presents the findings from the fiscal year (FY) 2010 annual review. The review was intended to answer the following questions regarding district-level funding: (1) how many funds were allocated to nutrition-specific activities in each district in FY 2010? (2) how many funds were allocated to twice-yearly VASD activities in each district in FY 2010?; and (3) how have VASD funding allocations changed over time?

\section{Methods \\ Data Collection}

It is recognised that according to the Lancet series, ${ }^{4}$ nutritionspecific activities are defined as interventions that address the immediate causes of malnutrition related to food intake, care practice, and disease. For the purpose of this review, these activities were defined as any fortification, supplementation, dietary/nutrition counselling or education activities, the treatment of malnutrition, and finally any other activities where the words 'nutrition' or 'food' were indicated.

Two trained VASD nutritionists were oriented on the methods for this review and then reviewed the CCHPs over the course of 2 weeks in October 2011. Two official final copies of all

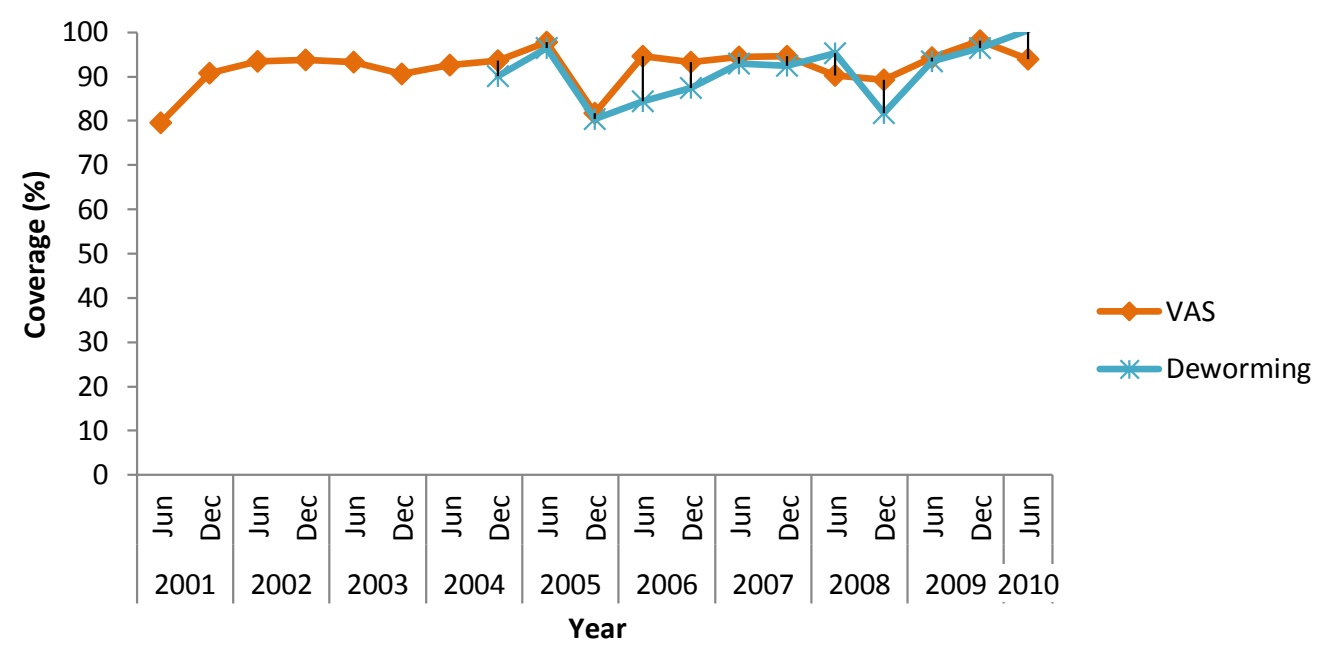

Figure 1. VAS Coverage in Children aged 6-59 Month in Tanzania. 


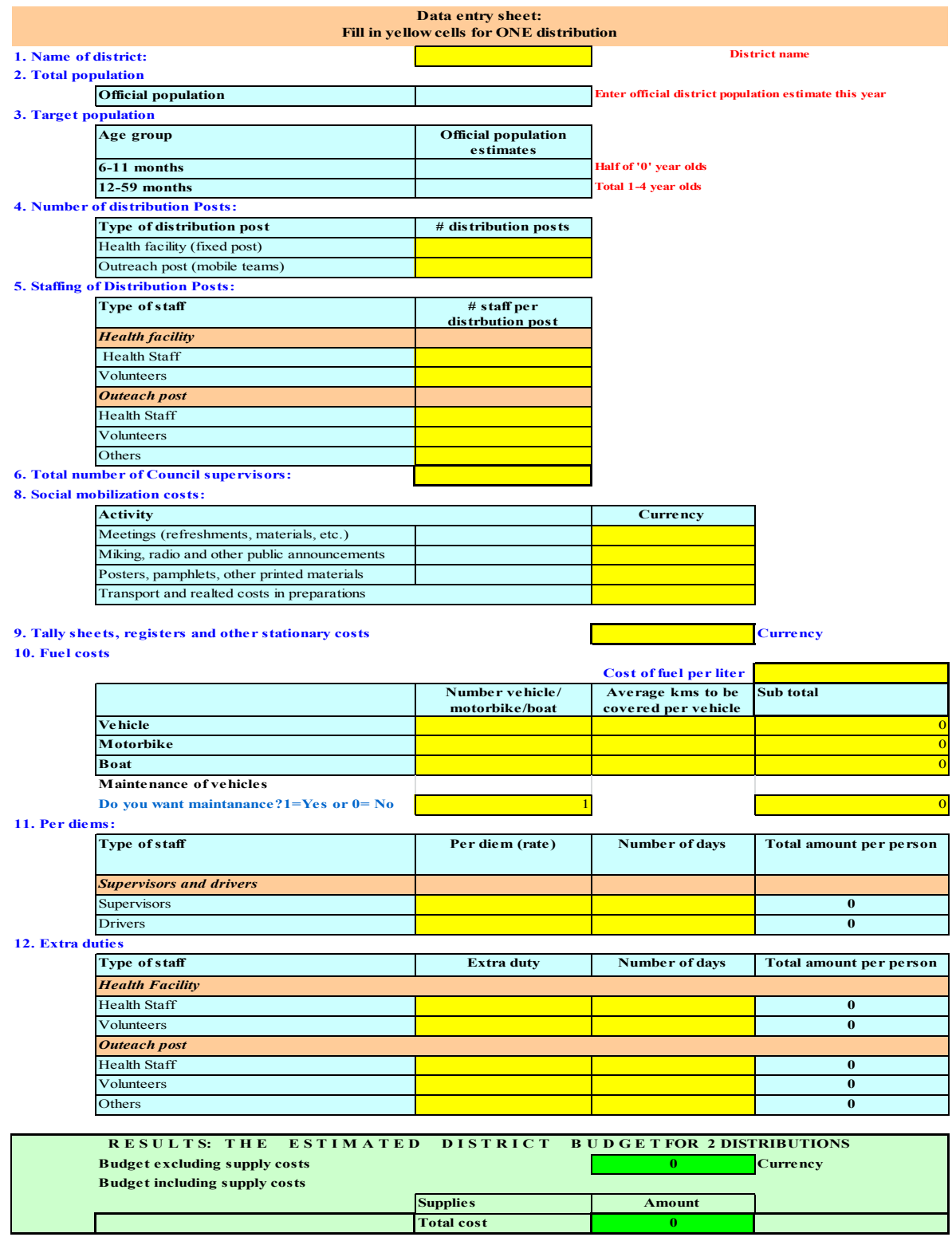

Figure 2. Planning and Budgeting Tool Template.

CCHPs from mainland Tanzania exist: one is stored in the offices of the Prime Minister Office Regional Administrative and Local Government (PMO-RALG) in Dodoma, Tanzania and the second is with the Ministry of Health and Social Welfare (MoHSW) in Dar es Salaam, Tanzania. As is done annually, permission to review one copy of the official CCHP from all districts $(\mathrm{n}=133)$ was obtained by the PMORALG office and copies were reviewed in situ at one of the two locations. Budget allocations were reviewed line by line and all identified activities and their allocated budget were recorded on a standardized data collection form. Visits were made to both offices since a complete set of CCHPs from each district was not found in either location alone. The CCHPs for each district reviewed were from FY 2010. Data from prior reviews (annually since FY 2005) were used for comparison and trend data. CCHP documents from all 133 mainland Tanzania districts were found and reviewed.

Data collected on integrated larger programs with nutrition subcomponents were not included in this analysis, unless there was a specific line item for a nutrition subcomponent, since it was not possible to determine the specific funding amount for nutrition. For example, although home-based care and prevention of mother to child transmission (PMTCT) programs have known but not quantifiable nutrition subcomponents, they were not included in this analysis.

\section{Analysis of Data}

From the data collection forms, budget allocations and the funding source for each activity and district were transferred into an Excel database formatted to mimic the data collection form.

In order to estimate the adequacy of the VASD funding levels, values were converted to "constant currency" to eliminate the impact of price changes or inflation. Constant currency is the currency value of several years expressed in terms of their value (purchasing power) in a single year (base year). To convert funds in Tanzanian Shilling (TZS) of any year to constant currency, the funding value was divided by the Tanzanian Consumer Price Index (CPI) of that year and multiplied the CPI of the chosen base year (2010). The 
constant TZS values were then expressed in US dollars using the year 2010 exchange rate. The CPI and average US\$-TZS exchange rate data were taken from the database of world development indicators. ${ }^{5}$

Adequacy was determined by an adjusted budget allocation per child. A costing study ${ }^{6}$ conducted using 2004 data indicated that the program specific annual costs for twice $=$ yearly provision of VASD was US $\$ 0.22$ per child (in 2004), which was used as a basis for determination of adequacy of funding allocations. Cost per child was calculated by dividing the total amount allocated in the CCHP for VASD by the target population of children aged 6-59 months during the corresponding year, using the official National Bureau of Statistics population projections for children 1-4 years and $50 \%$ of children under one year. Costs per child were also adjusted for constant currency.

\section{Results}

Nutrition-Specific District Funding

In FY 2010-2011, CCHPs for all 133 districts and councils in Tanzania were provided to the research team from the PMORALG Dodoma office. The total funds allocated for nutritionspecific activities in the CCHPs amounted to US $\$ 1.4$ million. VASD funding accounted for $92 \%$ of the total nutrition funds, or $\$ 1.3$ million (Table 1), of which $92.5 \%$ is from the HBF.

In all 133 CCHPs, budget allocations were made for VASD activities. In 10 districts (7.5\%), allocations were made for infant and young child feeding (IYCF) training, the next most-frequently budgeted nutrition activity (Table 2 ). A total of $24(18 \%)$ of the districts allocated some funds for nonVASD activities. Forty-two percent of non-VASD activities were funded by HBFs.

A total of 12 independent nutrition activities were budgeted across all districts, as shown in Figure 3. In terms of the highest absolute amount of funds, after VASD, training on
Essential Nutrition Action (ENA) (US\$30063), followed by orientation for health workers on IYCF feeding (US\$19637) were allocated funds. Iron-folate (IFA) supplements were the only nutrition commodities indicated in any of the budgets. This is interesting considering that although training on severe acute malnutrition (SAM) was budgeted, there were no allocations for supplies for management of SAM in health centers. It is also important to note that vitamin A capsules and mebendazole for deworming are provided as an in-kind donation to Tanzania via a DFATD/MI/UNICEF donation program and, therefore, are not included in the district budget allocations for VASD. As expected, before SUN commitments in the country, few nutrition-specific funds were allocated by district health teams in FY 2010 aside from VASD which had advocacy and facilitation at scale in country.

Vitamin A Supplementation and Deworming District Funding The total allocation for VASD was US\$1317129 $9^{[2]}$, or an average of US\$9903 per district for two rounds of VASD events. The amount allocated covers the recurrent costs related to implementation of VASD, including program staff and health worker per diems, transportation, and reporting and monitoring of the VASD events, excluding cost for the capsules and its distribution and any fixed health service costs.

\section{Trends in District Funding for Vitamin A Supplementation} Data from FY 2010 were compared against annual CCHP allocations for VASD since 2005 $5^{[3]}$. Between FY 2005 and FY 2010, allocation for VASD in district budgets increased from US $\$ 387$ thousand to US\$1.3 million (in constant dollars) as shown in Figure 4. When comparing trends in funding it is important to note that FY 2005 through FY 2007 represent a time when UNICEF was providing some direct support to some districts, therefore, the relatively low CCHP allocations in these earlier years do not necessarily reflect low VASD

Table 1. Budgeted Amounts (US\$) for VASD and Other Nutrition-Specific Activities in FY 2010 Tanzanian Comprehensive Council Health Plans

\begin{tabular}{llllll}
\hline Activity & Basket Fund & Block Grant & Council Fund & Other Funding & Total \\
\hline VASD & 1218426 & 87704 & 2129 & 1317129 & 8870 \\
Other nutrition-specifica activities & 45663 & - & 25677 & 37407 \\
Total & 1264090 & 87704 & 27806 & 108747 & 46276 \\
\hline
\end{tabular}

Abbreviations: VASD, vitamin A supplementation and deworming; FY, fiscal year.

${ }^{a}$ Nutrition specific was defined as any fortification, supplementation, dietary/nutrition counselling or education activities, the treatment of malnutrition, or any other activities where the words 'nutrition' or 'food' were indicated.

Table 2. Number and Percentage of Districts Funding Specific Nutrition Activities in Their CCHPs in FY 2010

\begin{tabular}{|c|c|c|c|}
\hline & Activity & No. of Districts Allocating Funds & Percent of Districts Aallocating Funds \\
\hline 1 & Twice-yearly VASD & 133 & 100.0 \\
\hline 2 & Training on IYCF & 10 & 7.5 \\
\hline 3 & Screening and management of SAM & 9 & 6.8 \\
\hline 4 & Purchasing iron and folic acid tables for antenatal care & 9 & 6.8 \\
\hline 5 & $\begin{array}{l}\text { Community mobilization on use of iodized salt and/or and iodine } \\
\text { inspection }\end{array}$ & 7 & 5.3 \\
\hline 6 & Providing health facility in-patients with food & 7 & 5.3 \\
\hline 7 & Training on the ENA & 6 & 4.5 \\
\hline 8 & Proving nutritional support to people living with HIV/AIDS & 2 & 1.5 \\
\hline 9 & Training and mobilization on food handling and food hygiene & 2 & 1.5 \\
\hline 10 & Nutrition surveys & 2 & 1.5 \\
\hline 11 & Providing food to women attending antenatal clinic & 1 & 0.8 \\
\hline 12 & Advocacy on emergency obstetric, nutrition, and child care & 1 & 0.8 \\
\hline
\end{tabular}

Abbreviations: ENA, essential nutrition actions; VASD, vitamin A supplementation and deworming; SAM, severe acute malnutrition; IYCF, infant and young child feeding. 


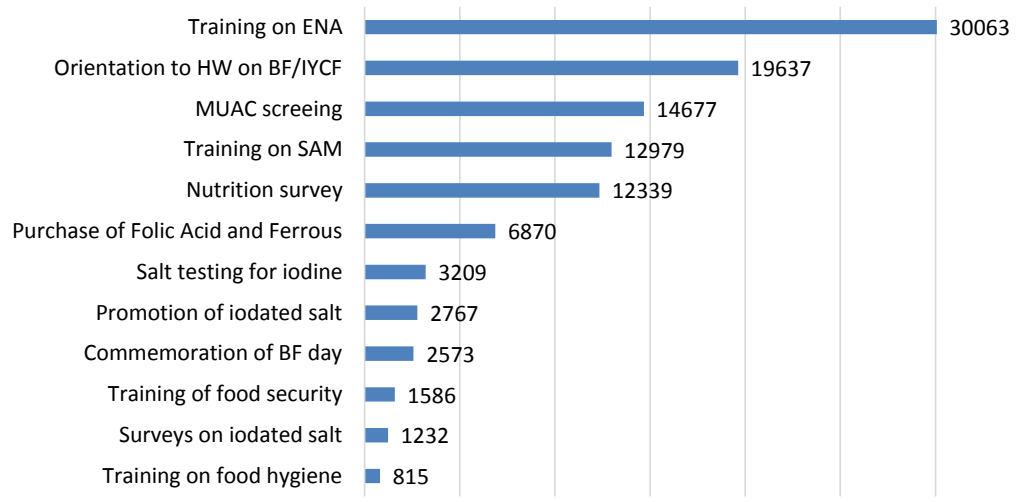

Figure 3. Comprehensive Council Health Plans (CCHP) funding allocation for nutrition activities other than vitamin A supplementation and deworming (VASD) in FY 2010 (US\$).

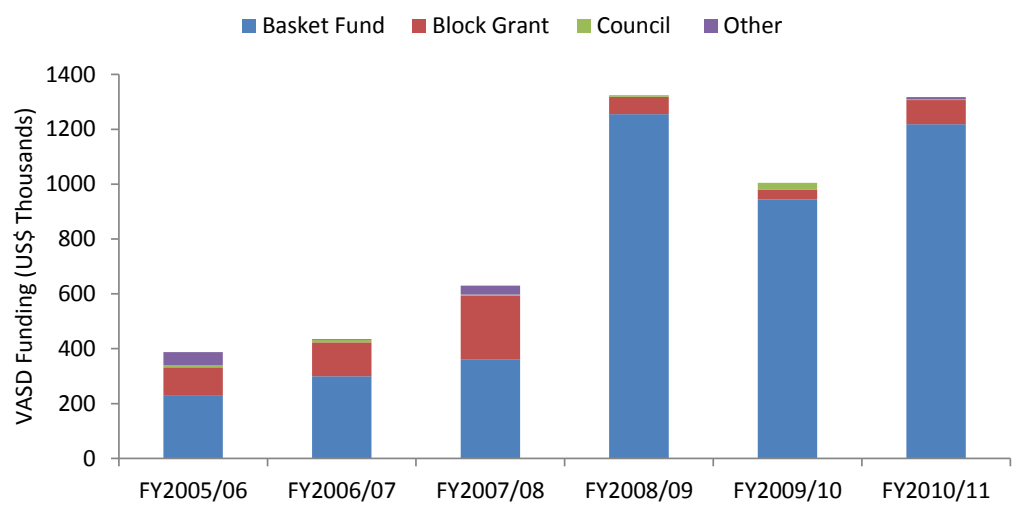

Figure 4. Comprehensive Council Health Plans (CCHP) Funding (in Constant Dollars) for vitamin A supplementation and deworming (VASD) by Source FY 2005-2010.

program funds. From FY 2008 onwards, UNICEF redirected VASD funds via the HBFs, thus a large increase is seen in CCHP allocations from then. In FY 2009, funding allocations dropped to roughly US\$1 million (in constant currency), and FY 2010 saw an increase in VASD allocations again to FY 2008 levels. A 2004 cost analysis ${ }^{6}$ found that $68 \%$ of programmatic costs for VASD implementation were for program personnel and volunteer allowances; and since government allowance rates from 2008 and 2010/2011 have not changed, there has been a relatively constant amount of funding since FY 2008. It was estimated $^{[4]}$ in 2004 that US $\$ 0.22$ per child was needed for sufficient implementation of VASD in Tanzania. In FY $2010 / 2011$ terms this would equate to US\$0.32 per child for program costs. Despite the increased trend on fund allocation as indicated in Figure 4, the percentage of districts allocating sufficient funds to implement VASD was just $21 \%$. This is not surprising given competing priorities and limited budget allocations for health. It is worth noting that Tanzania has not been able to achieve the Abuja declaration ${ }^{[5]}$ to set the health budget at $15 \%$ of the national budget ${ }^{7}$; the FY 2010 Health Sector budget represented $9 \%$ of the total government budget down from $11.3 \%$ in FY 2009. ${ }^{8}$

\section{Discussion}

The current district allocations cover approximately $2 \%$ of the estimated US\$66 million per year required in the National Nutrition Strategy Implementation Plan to implement key nutrition services. In order to fully fund the country's plan for scale-up of nutrition activities, a significantly higher level of funding needs to be allocated for service provision.

Aside from VASD, districts allocated very limited budgets for nutrition activities in FY 2010. There seemed to be a lack of consistency of activities across districts, as far as could be identified within the CCHPs, with no activities funded by all districts, except for VASD. For instance, in some cases one may find districts allocating funds to conduct staff training for SAM screening and management yet no budget was allocated for supplies related to management of SAM, unless those were not reported as noted in Musoma Municipal, Bunda, Tunduru, Kigoma district, Kibondo, Kasulu and Makete districts. The national VASD program and its national-level advocacy efforts applied in every district in the country may be key to that success.

All districts allocated funds for VASD in FY 2010/2011 indicating that the advocacy, tools provided and facilitation in the preceding years worked and was effective in securing funds for VASD. The effectiveness of the VASD program technical assistance was confirmed in an external VASD program assessment conducted in $2011^{9}$ that identified the PBT and the advocacy meetings as critical interventions for successful VASD programming.

Despite the VASD advocacy and facilitation work's success in ensuring all districts budget for the activity, only $21 \%$ of districts allocated 'sufficient funding' to implement the activity as estimated from a cost per child assumptions. Given the sustained and high coverage rates seen throughout this period (with some verification ${ }^{10}$ ), these cost per child estimates may not be a good indicator of sufficient funding. 
An updated cost analysis may be warranted as inflation alone may not account for real costs in VASD as compared to 2004. Based on the lessons learned from the VASD program, targeted advocacy (including planning tools) has resulted in sustained sub-national budget allocations for VASD activities over the last 5 years. To promote sustainable implementation of other nutrition interventions in Tanzania in the future, it is recommended that this approach be replicated for other interventions.

\section{Limitations of the Data Review}

A limitation of this review is that CCHPs provide information on funding allocations for specific activities, but do not necessarily represent the actual expenditures or true costs of an activity to achieve the targets. Therefore, there is a need for districts to follow-up, monitor and report the actual cost of implementing nutrition interventions, so that in subsequent years, allocations (or activities) can be fine-tuned and updated to reach the target. Another limitation is the inability of the CCHP to record nutrition-specific budget allocations particularly when allocations are embedded in larger programs that do not have disaggregated budgets. Finally, in reviewing the nutrition-specific activities, adequacy of funding was not quantifiable given the lack of information or tools on costing for these activities.

\section{Conclusion}

As demonstrated by CCHP allocations, VASD remained in FY 2010 the only subnationally planned and budgeted nutrition intervention implemented at scale in Tanzania. Tracking district budgets is a practical way of monitoring the impact of advocacy for nutrition on budget commitments.

As other nutrition interventions are challenged with national scale-up, it will be useful to expand the PBT tool to ensure adequate planning for key nutrition interventions.

Advocacy and facilitation efforts for nutrition programming will continue to include VASD but should also include other evidence-based nutrition interventions that have been shown to improve both child survival and nutritional status such as promoting adequate breastfeeding and dietary approaches to improve nutrition.

The lessons learned from the VASD program in Tanzania provide important insights into opportunities for scaling up other nutrition interventions in the country, especially interventions that must be planned, budgeted and implemented by the districts. This integration should ensure that district health plans incorporate the endorsed National Nutrition Strategy Implementation Plan activities in as much as possible so that Tanzania is able to meet the SUN commitments it set aside in 2011. And ultimately, more funds need to be committed to nutrition activities in Tanzania. Other countries can learn from Tanzania's district advocacy approach and the technical assistance for planning and budgeting as they switch to health basket funding and/or decentralized systems.

\section{Acknowledgements}

The authors would like to thank Wessy Meghi and Eunice Rwiza for their work during data compilation. We also appreciate the support of the Ministry of Health staff and the Prime Minister's Office-Regional Administrative and Local Government where the council plans and budget were obtained and reviewed.

Ethical issues

None. This assessment is part of routine program activities and permission for document review was provided by the respective and relevant government authorities.

\section{Authors' contributions}

$\mathrm{ML}$ was involved in analysis, interpretation, and writing of the paper. TM was involved in the study design, data collection, and interpretation. RS was involved in the study design and result review. MI was involved in the data analysis, review, and editing of the manuscript. FM was involved in study design and data collection. HK was involved in data interpretation and review. CND was involved in the study design, overall supervision, data interpretation, and writing. All authors were involved in the review and editing of the manuscript drafts and decision to submit the paper for publication.

Competing interests

Authors declare that they have no competing interests.

\section{Endnotes}

[1] In this manuscript, 'nutrition services' is the term used within the Tanzania National Nutrition Strategy's Implementation Plan line item for 'service delivery' of nutrition programs.

[2] Using 2010 exchange rate from actual allocations made in Tanzanian Shillings.

[3] HKI (2009) Comprehensive Council Health Plan Review for VAS Activities. HKI Tanzania, Dar es Salaam database.

[4] Costing study citation.

[5] Agreed in April 2001 by the heads of the African Union.

\section{Authors' affiliations}

${ }^{1}$ Helen Keller International, Dar es Salaam, Tanzania. ${ }^{2}$ United States Agency for International Development (USAID), Dar es Salaam, Tanzania. ${ }^{3}$ Tanzania Food and Nutrition Centre, Dar es Salaam, Tanzania.

\section{References}

1. Tanzania Food and Nutrition Centre (TFNC). Landscape Analysis of countries' readness to accelerate action in nutrition. Tanzania assessment for scalling up nutrition. TFNC report; 2012

2. Ministry of Health and Social Welfare (MoHSW). Comprehensive Council Health Management Planning Guideline. The United Republic of Tanzania: Ministry of Health; 2010.

3. Masanja $H$, de Savigny $D$, Smithson $P$, et al. Child survival gains in Tanzania: analysis of data from demographic and health surveys. Lancet. 2008;371(9620):1276-1283. doi:10.1016/ S0140-6736(08)60562-0

4. Ruel MT, Alderman H; Maternal and Child Nutrition Study Group. Nutrition sensitive interventions and programs; how can they help to accelerate progress in improving martenal and child nutrition. Lancet. 2013;382(9891):536-551. doi:10.1016/S01406736(13)60843-0.

5. Worldbank. World Development Indicators. http://data.worldbank. org/data-catalog/world-development-indicators

6. Rassas BM, Mulokozi G, Mugyabuso J, Lukmanji Z, Ruhiye D, Modaha F. Cost Analysis of the National Twice-Yearly Vitamin A Supplementation Program in Tanzania. Arlington, Virginia; 2005.

7. World Health Organization (WHO). Abuja Declaration: Ten years On. http://www.who.int/healthsystems/publications/abuja_ report_aug_2011.pdf. Accessed February 2015. Published 2001.

8. Sikika. 2010/2011 Health Sector Budget Analysis. Dar es Salaam, Tanzania: Sikika; 2011.

9. Mullins J, Ehrlich L. Assessment of the National Vitamin A Supplementation and De-worming Program in Tanzania. WellShare; 2011.

10. Nyhus Dhillon C, Subramaniam H, Mulokozi G, Rambeloson Z, Klemm R. Overestimation of vitamin a supplementation coverage from district tally sheets demonstrates importance of population-based surveys for program improvement: lessons from Tanzania. PLoS One. 2013;8(3):e58629. doi:10.1371/ journal.pone.0058629 